\title{
ON THE LOG-CONCAVITY OF HILBERT SERIES OF VERONESE SUBRINGS AND EHRHART SERIES
}

\author{
MATTHIAS BECK AND ALAN STAPLEDON
}

\begin{abstract}
For every positive integer $n$, consider the linear operator $\mathrm{U}_{n}$ on polynomials of degree at most $d$ with integer coefficients defined as follows: if we write $\frac{h(t)}{(1-t)^{d+1}}=\sum_{m \geq 0} g(m) t^{m}$, for some polynomial $g(m)$ with rational coefficients, then $\frac{\mathrm{U}_{n} h(t)}{(1-t)^{d+1}}=\sum_{m \geq 0} g(n m) t^{m}$. We show that there exists a positive integer $n_{d}$, depending only on $d$, such that if $h(t)$ is a polynomial of degree at most $d$ with nonnegative integer coefficients and $h(0)=1$, then for $n \geq n_{d}, \mathrm{U}_{n} h(t)$ has simple, real, negative roots and positive, strictly log concave and strictly unimodal coefficients. Applications are given to Ehrhart $\delta$-polynomials and unimodular triangulations of dilations of lattice polytopes, as well as Hilbert series of Veronese subrings of Cohen-Macauley graded rings.
\end{abstract}

\section{INTRODUCTION}

Fix a positive integer $d$. If $h(t)=h_{0}+h_{1} t+\cdots+h_{d} t^{d}$ is a nonzero polynomial of degree at most $d$ with nonnegative integer coefficients and $h_{0}=1$, then

$$
\frac{h(t)}{(1-t)^{d+1}}=\sum_{m \geq 0} g(m) t^{m}
$$

where $g(m)=\sum_{i=0}^{d} h_{i}\left(\begin{array}{c}m+d-i \\ d\end{array}\right)$ is a polynomial of degree $d$ with rational coefficients. For every positive integer $n$, define $\mathrm{U}_{n} h(t)$ to be the polynomial of degree at most $d$ with integer coefficients satisfying

$$
\frac{\mathrm{U}_{n} h(t)}{(1-t)^{d+1}}=\sum_{m \geq 0} g(n m) t^{m}
$$

and write $\mathrm{U}_{n} h(t)=h_{0}(n)+h_{1}(n) t+\cdots+h_{d}(n) t^{d}$. The (Hecke) operator $\mathrm{U}_{n}$ was studied by Gil and Robins in a more general setting [15] and more recently by Brenti and Welker [6]. The goal of this paper is to show that there exists a positive integer $n_{d}$, depending only on $d$, such that $\mathrm{U}_{n} h(t)$ is well-behaved, in a sense to be defined, for $n \geq n_{d}$.

Our main motivating example comes from the theory of lattice point enumeration of polytopes. More specifically, let $N$ be a lattice of rank $n$ and set $N_{\mathbb{R}}:=N \otimes_{\mathbb{Z}} \mathbb{R}$. A lattice polytope $P \subset N_{\mathbb{R}}$ is the convex hull of finitely many points in $N$. Fix a $d$-dimensional lattice polytope $P \subset N_{\mathbb{R}}$ and, for each positive integer $m$, let $f_{P}(m):=\#(m P \cap N)$ denote the number of lattice points in the $m$ 'th dilate of $P$. A famous theorem of

Date: 13 October 2008.

2000 Mathematics Subject Classification. 05A15; 13C14, 52B20.

The authors would like to thank Alexander Barvinok, Jesus De Loera, Sergey Fomin, Joseph Gubeladze, Mircea Mustaţă, Sam Payne, John Stembridge, Volkmar Welker, and an anonymous referee for useful discussions and helpful suggestions. The first author was partially supported by the NSF (research grant DMS-0810105), and the second author was partially supported by an Eleanor Sophia Wood travelling scholarship from the University of Sydney. 
Ehrhart 13 asserts that $f_{P}(m)$ is a polynomial in $m$ of degree $d$, called the Ehrhart polynomial of $P$, and $f_{P}(0)=1$. Equivalently, the generating series of $f_{P}(m)$ can be written in the form

$$
\frac{\delta_{P}(t)}{(1-t)^{d+1}}=\sum_{m \geq 0} f_{P}(m) t^{m}
$$

where $\delta_{P}(t)=\delta_{0}+\delta_{1} t+\cdots+\delta_{d} t^{d}$ is a polynomial of degree at most $d$ with integer coefficients, called the $\delta$-polynomial of $P$, and $\delta_{0}=1$. We call $\left(\delta_{0}, \delta_{1}, \ldots, \delta_{d}\right)$ the (Ehrhart) $\delta$-vector of $P$; alternative names in the literature include Ehrhart $h$-vector and $h^{*}$-vector of $P$. Stanley proved that the coefficients $\delta_{i}$ are nonnegative 22. In this case, $\mathrm{U}_{n} \delta_{P}(t)=\delta_{n P}(t)$ and we write $\delta_{n P}(t)=\delta_{0}(n)+\delta_{1}(n) t+\cdots+\delta_{d}(n) t^{d}$.

More generally, let $R=\oplus_{i \geq 0} R_{i}$ be a graded ring of dimension $d+1$ and assume that $R_{0}=k$ is a field and that $R$ is finitely generated over $R_{0}$. The $n$ 'th Veronese subring of $R$ is the graded $\operatorname{ring} R^{\langle n\rangle}=\oplus_{i \geq 0} R_{i n}$. The behaviour of Veronese subrings for large $n$ has been studied by Backelin [2] and Eisenbud, Reeves and Totaro [14. The Hilbert function of $R$ is defined by $H(R, m)=\operatorname{dim}_{k} R_{m}$, for each nonnegative integer $m$, and by a theorem of Hilbert [11, Theorem 4.1.3], $H(R, m)$ is a polynomial in $m$ of degree $d$ for $m$ sufficiently large. In fact, $H(R, m)$ is a polynomial for $m>a(R)$, where $a(R)$ is the a-invariant of $R$ and is defined in terms of the local cohomology of $R$ [11, Section 3.6]. Observe that this implies that $H\left(R^{\langle n\rangle}, m\right)$ is a polynomial in $m$ of degree $d$ for $n>a(R)$. Assume that $R$ is Cohen-Macauley and that $R$ is a finite module over the $k$-subalgebra of $R$ generated by $R_{1}$. If $H(R, m)$ is a polynomial in $m$ then it can be seen as in [11, Corollary 4.1.10] that

$$
\frac{h_{0}+h_{1} t+\cdots+h_{d} t^{d}}{(1-t)^{d+1}}=\sum_{m \geq 0} H(R, m) t^{m},
$$

for some nonnegative integers $h_{i}$, with $h_{0}=1$. For every positive integer $n$, the numerator of the generating series of $H\left(R^{\langle n\rangle}, m\right)$ has the form $(1-t)^{d+1} \sum_{m \geq 0} H\left(R^{\langle n\rangle}, m\right) t^{m}=\mathrm{U}_{n}\left(h_{0}+h_{1} t+\cdots+h_{d} t^{d}\right)$. Returning to our previous example, if $N^{\prime}=N \times \mathbb{Z}$ and $\sigma$ denotes the cone over $P \times\{1\}$ in $N_{\mathbb{R}}^{\prime}$, then the semigroup algebra $R=k\left[\sigma \cap N^{\prime}\right]$ is graded by the projection $u: N^{\prime} \rightarrow \mathbb{Z}$ and satisfies the above assumptions [11, Theorem 6.3.5]. In this case, $H(R, m)=f_{P}(m)$ is the Ehrhart polynomial of $P$ and $\mathrm{U}_{n}\left(h_{0}+h_{1} t+\cdots+h_{d} t^{d}\right)=\delta_{n P}(t)$.

A sequence of positive integers $\left(a_{0}, \ldots, a_{d}\right)$ is strictly log concave if $a_{i}^{2}>a_{i-1} a_{i+1}$ for $1 \leq i \leq d-1$ and is strictly unimodal if $a_{0}<a_{1}<\cdots<a_{j}$ and $a_{j+1}>a_{j+2}>\cdots>a_{d}$ for some $0 \leq j \leq d$. One easily verifies that if $\left(a_{0}, \ldots, a_{d}\right)$ is strictly $\log$ concave then it is strictly unimodal. An induction argument implies that if the polynomial $a_{0}+a_{1} t+\cdots+a_{d} t^{d}$ has negative real roots then the sequence $\left(a_{0}, \ldots, a_{d}\right)$ is strictly log concave and hence strictly unimodal. Brenti and Welker recently proved the following theorem [6. Theorem 1.4].

Theorem 1.1 (Brenti-Welker). For any positive integer $d$, there exist real numbers $\alpha_{1}<\alpha_{2}<\cdots<$ $\alpha_{d-1}<\alpha_{d}=0$ such that, if $h(t)=h_{0}+h_{1} t+\cdots+h_{d} t^{d}$ is a polynomial of degree at most $d$ with nonnegative integer coefficients and $h_{0}=1$, then for $n$ sufficiently large, $\mathrm{U}_{n} h(t)$ has negative real roots $\beta_{1}(n)<\beta_{2}(n)<\cdots<\beta_{d-1}(n)<\beta_{d}(n)<0$ and $\beta_{i}(n) \rightarrow \alpha_{i}$ as $n \rightarrow \infty 1$.

Let $w=\left(w_{1}, \ldots, w_{d}\right)$ be a permutation of $d$ elements. A descent of $w$ is an index $1 \leq j \leq d-1$ such that $w_{j+1}<w_{j}$. If $A(d, i)$ denotes the number of permutations of $d$ elements with $i-1$ descents, then the

\footnotetext{
${ }^{1}$ In [6], the notation $\beta_{i}(n)$ is used for the reciprocals of the roots.
} 
polynomial $A_{d}(t)=\sum_{i=1}^{d} A(d, i) t^{i}$ is called an Eulerian polynomial and the roots of $\frac{A_{d}(t)}{t}$ are simple, real and strictly negative [12, p. 292, Exercise 3]. We are ready to state our main result and emphasise that the real content of Theorem 1.2 is the statement that the constants $m_{d}$ and $n_{d}$ below only depend on $d$ (and not on $h(t))$.

Theorem 1.2. Fix a positive integer $d$ and let $\rho_{1}<\rho_{2}<\cdots<\rho_{d}=0$ denote the roots of the Eulerian polynomial $A_{d}(t)$. There exist positive integers $m_{d}$ and $n_{d}$ such that, if $h(t)$ is a polynomial of degree at most $d$ with nonnegative integer coefficients and $h_{0}=1$, then for $n \geq n_{d}, \mathrm{U}_{n} h(t)$ has negative real roots $\beta_{1}(n)<\beta_{2}(n)<\cdots<\beta_{d-1}(n)<\beta_{d}(n)<0$ with $\beta_{i}(n) \rightarrow \rho_{i}$ as $n \rightarrow \infty$, and the coefficients of $\mathrm{U}_{n} h(t)$ are positive, strictly log concave, and satisfy $h_{i}(n)<m_{d} h_{d}(n)$ for $0 \leq i \leq d$. Furthermore, we may choose $n_{d}$ such that, if additionally $h_{0}+\cdots+h_{i+1} \geq h_{d}+\cdots+h_{d-i}$ for $0 \leq i \leq\left\lfloor\frac{d}{2}\right\rfloor-1$, then

$$
h_{0}=h_{0}(n)<h_{d}(n)<h_{1}(n)<\cdots<h_{i}(n)<h_{d-i}(n)<h_{i+1}(n)<\cdots<h_{\left\lfloor\frac{d+1}{2}\right\rfloor}(n)<m_{d} h_{d}(n) .
$$

If $h(t)=\delta_{P}(t)$ then assumptions of the above theorem hold by a result of Hibi [16, and we deduce the following corollary.

Corollary 1.3. Fix a positive integer $d$ and let $\rho_{1}<\rho_{2}<\cdots<\rho_{d}=0$ denote the roots of the Eulerian polynomial $A_{d}(t)$. There exists positive integers $m_{d}$ and $n_{d}$ such that, if $P$ is a d-dimensional lattice polytope and $n \geq n_{d}$, then $\delta_{n P}(t)$ has negative real roots $\beta_{1}(n)<\beta_{2}(n)<\cdots<\beta_{d-1}(n)<\beta_{d}(n)<0$ with $\beta_{i}(n) \rightarrow \rho_{i}$ as $n \rightarrow \infty$, and the coefficients of $\delta_{n P}(t)$ are positive, strictly log concave, and satisfy

$$
1=\delta_{0}(n)<\delta_{d}(n)<\delta_{1}(n)<\cdots<\delta_{i}(n)<\delta_{d-i}(n)<\delta_{i+1}(n)<\cdots<\delta_{\left\lfloor\frac{d+1}{2}\right\rfloor}(n)<m_{d} \delta_{d}(n) .
$$

We also have the following application to Veronese subrings of graded rings.

Corollary 1.4. Fix a positive integer $d$ and let $\rho_{1}<\rho_{2}<\cdots<\rho_{d}=0$ denote the roots of the Eulerian polynomial $A_{d}(t)$. There exists positive integers $m_{d}$ and $n_{d}$ such that, if $R=\oplus_{i \geq 0} R_{i}$ is a finitely generated graded ring over a field $R_{0}=k$, which is Cohen-Macauley and module finite over the $k$-subalgebra of $R$ generated by $R_{1}$, and if the Hilbert function $H(R, m)$ is a polynomial in $m$ and we write

$$
\frac{\mathrm{U}_{n} h(t)}{(1-t)^{d+1}}=\sum_{m \geq 0} H\left(R^{\langle n\rangle}, m\right) t^{m}
$$

for each positive integer $n$, then for $n \geq n_{d}, \mathrm{U}_{n} h(t)$ has negative real roots $\beta_{1}(n)<\beta_{2}(n)<\cdots<\beta_{d-1}(n)<$ $\beta_{d}(n)<0$ with $\beta_{i}(n) \rightarrow \rho_{i}$ as $n \rightarrow \infty$, and the coefficients of $\mathrm{U}_{n} h(t)$ are positive, strictly log concave, and satisfy $h_{i}(n)<m_{d} h_{d}(n)$ for $0 \leq i \leq d$.

It is an open problem to determine the optimal choices for the integers $m_{d}$ and $n_{d}$ in Theorem 1.2 and Corollaries 1.3 and 1.4. In this direction, we show that for any positive integer $d$ and $n \geq d$, if $h(t)$ satisfies certain inequalities, then $h_{i+1}(n)>h_{d-i}(n)$ for $i=0, \ldots,\left\lfloor\frac{d}{2}\right\rfloor-1$ (Theorem 4.6). In particular, this holds when $h(t)=\delta_{P}(t)$ (Example 4.7).

We now explain our original motivation for this paper. A triangulation $\tau$ of the polytope $P$ with vertices in $N$ is unimodular if for any simplex of $\tau$ with vertices $v_{0}, v_{1}, \ldots, v_{d}$, the vectors $v_{1}-v_{0}, \ldots, v_{d}-v_{0}$ form a basis of $N$. While every lattice polytope can be triangulated into lattice simplices, it is far from true that every lattice polytope admits a unimodular triangulation (for an easy example, consider the convex 
hull of $(1,0,0),(0,1,0),(0,0,1)$, and $(1,1,1))$. The following theorem, however, says that we can obtain a unimodular triangulation if we allow our polytope to be dilated.

Theorem 1.5 (Knudsen-Mumford-Waterman [18]). For every lattice polytope $P$, there exists an integer $n$ such that $n P$ admits a regular unimodular triangulation.

For a general reference on triangulations, including regular ones, see 20. If $P$ admits a unimodular triangulation, then every multiple $n P$ admits such a triangulation (this follows from the general theory of Knudsen-Mumford triangulations; see [8, Remark 3.19]). Thus Theorem 1.5 implies that $k n P$ admits a unimodular triangulation for $k \in \mathbb{Z}_{>0}$. There are several conjectured stronger versions of Theorem 1.5 (see, for example, [9, 10]):

Conjecture 1.6. (a) For every lattice polytope $P$, there exists an integer $m$ such that $n P$ admits a regular unimodular triangulation for $n \geq m$.

(b) For every $d \in \mathbb{Z}_{>0}$, there exists an integer $n_{d}$ such that, if $P$ is a d-dimensional lattice polytope, then $n_{d} P$ admits a regular unimodular triangulation.

(c) For every $d \in \mathbb{Z}_{>0}$, there exists an integer $n_{d}$ such that, if $P$ is a d-dimensional lattice polytope, then $n P$ admits a regular unimodular triangulation for $n \geq n_{d}$.

When $d=1$ or 2, every lattice polytope has a unimodular triangulation. For $d=3$, Conjecture 1.6(b) holds with $n_{3}=4$ [17.

Conjecture [1.6 was the first motivation for our paper, and the following result [1, Theorem 1.3] was the second.

Theorem 1.7 (Athanasiadis-Hibi-Stanley). If a d-dimensional lattice polytope $P$ admits a regular unimodular triangulation, then the $\delta$-vector of $P$ satisfies

(a) $\delta_{i+1} \geq \delta_{d-i}$ for $0 \leq i \leq\left\lfloor\frac{d}{2}\right\rfloor-1$,

(b) $\delta_{\left\lfloor\frac{d+1}{2}\right\rfloor} \geq \delta_{\left\lfloor\frac{d+1}{2}\right\rfloor+1} \geq \cdots \geq \delta_{d-1} \geq \delta_{d}$,

(c) $\delta_{i} \leq\left(\begin{array}{c}\delta_{1}+i-1 \\ i\end{array}\right)$ for $0 \leq i \leq d$.

In particular, if the $\delta$-vector of $P$ is symmetric and $P$ admits a regular unimodular triangulation, then the $\delta$-vector is unimodal.

In fact, the first inequality in the above theorem holds under the weaker assumption that the boundary of $P$ admits a regular unimodular triangulation [23, Theorem 2.20]. There are (many) lattice polytopes for which some of the inequalities of Theorem 1.7 fail and one may hope to use Theorem 1.7 to construct a counter-example to Conjecture 1.6. However, a consequence of Corollary 1.3 and its proof is that this approach can not possibly work. More precisely, one can show that there exists a positive integer $n_{d}$ such that if $n \geq n_{d}$, then the inequalities in Theorem 1.7 hold for $n P$.

We end the introduction with a brief outline of the contents of the paper. In Section 2, we develop some inequalities between the coefficients of polynomials with certain properties, and we remark that Theorem 2.11 might be interesting in its own right - it asserts that we can bound roughly half the coefficients of an Ehrhart polynomial in terms of the dimension of $P$ and the surface area of $P$. In Section 3, we express $h_{i}(n)$ 
as a sum of Eulerian polynomials for $1 \leq i \leq d$ and use this description to establish our main results. In Section 4 , we consider bounds for $n_{d}$ and prove the aforementioned Theorem 4.6. We conclude in Section 5 with a conjecture on Ehrhart $\delta$-vectors.

\section{Inequalities Between Coefficients of Polynomials}

Our setup in this section will be slightly more general than the one in the introduction. We fix the following notation throughout the paper. Let $h(t)=h_{0}+h_{1} t+\cdots+h_{d+1} t^{d+1}$ be a nonzero polynomial of degree at most $d+1$ with integer coefficients, and write

$$
h_{0}+\sum_{m \geq 1} g(m) t^{m}=\frac{h(t)}{(1-t)^{d+1}},
$$

where $g(m)=\sum_{i=0}^{d+1} h_{i}\left(\begin{array}{c}m+d-i \\ d\end{array}\right)$ is a polynomial with rational coefficients. We write $g(t)=g_{d} t^{d}+g_{d-1} t^{d-1}+$ $\cdots+g_{0}$ and will assume that $g_{d}=\frac{\sum_{i=0}^{d+1} h_{i}}{d !}$ is positive and hence bounded below by $\frac{1}{d !}$. One can verify that $h_{d+1}=(-1)^{d}\left(g(0)-h_{0}\right)$ and we will often assume that $h(t)$ is a polynomial of degree at most $d$, in which case $g(0)=h_{0}$.

Example 2.1. A lattice complex $K$ in a lattice $N$ is a simplicial complex in $N_{\mathbb{R}}$ whose vertices lie in $N$. A lattice complex is pure of dimension $r$ if all its maximal simplices have dimension $r$. Let $K$ be a pure lattice complex of dimension $r$ and, for each positive integer $m$, let $f_{K}(m):=\#(m K \cap N)$ denote the number of lattice points in the $m$ 'th dilate of $K$. Ehrhart's theorem implies that $f_{K}(m)$ is a polynomial in $m$ of degree $r$. If we write $1+\sum_{m \geq 1} f_{K}(m) t^{m}=\frac{\delta_{K}(t)}{(1-t)^{r+1}}$, then Betke and McMullen [5] showed that $\delta_{K}(t)$ has nonnegative coefficients if $K$ is homeomorphic to a ball or a sphere. Moreover, $\delta_{K}(t)$ has degree at most $d$ when $K$ is homeomorphic to a ball and the coefficients of $\delta_{K}(t)$ are symmetric when $K$ is homeomorphic to a sphere. For example, a $d$-dimensional lattice polytope $P$ is homeomorphic to a $d$-ball and can be given the structure of a pure lattice complex of dimension $d$. Its boundary $\partial P$ is homeomorphic to a $(d-1)$-sphere and can be given the structure of a pure lattice complex of dimension $d-1$.

The following inequalities and their proof are a slight generalisation of [5, Theorem 6]. Recall that the Stirling number $S_{i}(d)$ of the first kind is the coefficient of $t^{i}$ in $\prod_{j=0}^{d-1}(t-j)$; note that $(-1)^{d-i} S_{i}(d)>0$ for $i \geq 1$.

Theorem 2.2 (Betke-McMullen). With the notation of (11), if $h_{i} \geq 0$ for $0 \leq i \leq d+1$, then for any $1 \leq r \leq d-1$

$$
g_{r} \leq(-1)^{d-r} S_{r}(d) g_{d}+\frac{(-1)^{d-r-1} h_{0} S_{r+1}(d)}{(d-1) !}
$$

Proof. By definition, $g_{r}=\sum_{i=0}^{d+1} h_{i}\left(\begin{array}{c}m+d-i \\ d\end{array}\right)_{r}$, where $\left(\begin{array}{c}m+d-i \\ d\end{array}\right)_{r}$ denotes the coefficient of $m^{r}$ in $\left(\begin{array}{c}m+d-i \\ d\end{array}\right)$. Observe that $\left(\begin{array}{c}m+d \\ d\end{array}\right)_{r} \geq\left(\begin{array}{c}m+d-1 \\ d\end{array}\right)_{r} \geq\left(\begin{array}{c}m+d-i \\ d\end{array}\right)_{r} \quad$ for $\quad 2 \leq i \leq d+1$, and hence, by the nonnegativity of the $h_{i}, g_{r} \leq h_{0}\left(\begin{array}{c}m+d \\ d\end{array}\right)_{r}+\sum_{i=1}^{d+1} h_{i}\left(\begin{array}{c}m+d-1 \\ d\end{array}\right)_{r}$. Using the fact that $d ! g_{d}=\sum_{i=0}^{d+1} h_{i}$ and applying a binomial identity, we get $g_{r} \leq h_{0}\left(\begin{array}{c}m+d-1 \\ d-1\end{array}\right)_{r}+d ! g_{d}\left(\begin{array}{c}m+d-1 \\ d\end{array}\right)_{r}$. Observing that $\left(\begin{array}{c}m+d-1 \\ d-1\end{array}\right)_{r}$ is the coefficient of $m^{r+1}$ in $\frac{\prod_{j=0}^{d-1}(m+j)}{(d-1) !}$, which is the coefficient of $(-m)^{r+1}$ in $\frac{(-1)^{d} \prod_{j=0}^{d-1}(m-j)}{(d-1) !}$, we conclude that $\left(\begin{array}{c}m+d-1 \\ d-1\end{array}\right)_{r}=\frac{(-1)^{d-r-1} S_{r+1}(d)}{(d-1) !}$. Similarly, one can verify that $\left(\begin{array}{c}m+d-1 \\ d\end{array}\right)_{r}=\frac{(-1)^{d-r} S_{r}(d)}{d !}$ and the result follows. 
Example 2.3. If $P$ is a $d$-dimensional lattice polytope, denote its Ehrhart polynomial by $f_{P}(m)=c_{d} m^{d}+$ $c_{d-1} m^{d-1}+\cdots+c_{0}$. Basic facts of Ehrhart theory (see, e.g., 4]) imply that $c_{d}$ is the normalised volume of $P$ and $c_{d-1}$ is half the normalised surface area of $P$. In this case, $h(t)=\delta_{P}(t)$ is the Ehrhart $\delta$-polynomial of $P$ and $\delta_{0}=1$. Since the coefficients of $\delta_{P}(t)$ are nonnegative [22, Theorem 2.2 implies that the coefficients $c_{i}$ can be bounded in terms of $d$ and the volume of $P$ (a fact that follows also, e.g., from [19]).

We can strengthen these inequalities if we put further restrictions on the coefficients $h_{i}$. We will need the following lemmas, the first of which is motivated by similar results in 23 .

Lemma 2.4. A polynomial $h(t)=h_{0}+h_{1} t+\cdots+h_{d+1} t^{d+1}$ with integer coefficients has a unique decomposition $h(t)=a(t)+b(t)$, where $a(t)$ and $b(t)$ are polynomials with integer coefficients satisfying $a(t)=t^{d} a\left(\frac{1}{t}\right)$ and $b(t)=t^{d+1} b\left(\frac{1}{t}\right)$.

Proof. Let $a_{i}$ and $b_{i}$ denote the coefficients of $t^{i}$ in $a(t)$ and $b(t)$ respectively, and set

$$
\begin{gathered}
a_{i}=h_{0}+\cdots+h_{i}-h_{d+1}-\cdots-h_{d+1-i}, \\
b_{i}=-h_{0}-\cdots-h_{i-1}+h_{d+1}+\cdots+h_{d+1-i} .
\end{gathered}
$$

We see that $h(t)=a(t)+b(t)$ and

$$
\begin{gathered}
a_{i}-a_{d-i}=h_{0}+\cdots+h_{i}-h_{d+1}-\cdots-h_{d-i+1}-h_{0}-\cdots-h_{d-i}+h_{d+1}+\cdots+h_{i+1}=0, \\
b_{i}-b_{d+1-i}=-h_{0}-\cdots-h_{i-1}+h_{d+1}+\cdots+h_{d+1-i}+h_{0}+\cdots+h_{d-i}-h_{d+1}-\cdots-h_{i}=0,
\end{gathered}
$$

for $0 \leq i \leq d+1$. Hence we obtain our desired decomposition and one easily verifies the uniqueness assertion.

Remark 2.5. Alternatively, to prove the above lemma, one can check that $a(t)=\frac{h(t)-t^{d+1} h\left(t^{-1}\right)}{1-t}$ and $b(t)=$ $\frac{-t h(t)+t^{d+1} h\left(t^{-1}\right)}{1-t}$.

Remark 2.6. It follows from (2) that $a(t)$ is nonzero with nonnegative integer coefficients if and only if $h_{0}+\cdots+h_{i} \leq h_{d+1}+\cdots+h_{d+1-i}$ for $0 \leq i \leq\left\lfloor\frac{d}{2}\right\rfloor$, with at least one of these inequalities strict. The coefficients of $a(t)$ are positive if and only if each of the above inequalities are strict. Since $a_{i+1}-a_{i}=h_{i+1}-h_{d-i}$, we see that the coefficients of $a(t)$ are unimodal (resp. strictly unimodal) if and only if $h_{i+1} \geq h_{d-i}$ (resp. $h_{i+1}>h_{d-i}$ ) for $0 \leq i \leq\left\lfloor\frac{d}{2}\right\rfloor-1$.

Example 2.7. If $P$ is a $d$-dimensional lattice polytope and we write $\delta_{P}(t)=a(t)+b(t)$ as in Lemma 2.4 then [23. Theorem 2.14] implies that $1=a_{0} \leq a_{1} \leq a_{i}$ for $2 \leq i \leq d-1$. In particular, $a(t)$ has degree $d$ and positive integer coefficients. We have $\delta_{0}=1>\delta_{d+1}=0$ and, by the above remark, $\delta_{0}+\cdots+\delta_{i} \leq \delta_{d}+\cdots+\delta_{d+1-i}$, for $1 \leq i \leq\left\lfloor\frac{d}{2}\right\rfloor$. The latter inequalities were proved by Hibi [16], and the fact that the coefficients of $a(t)$ are positive implies that all of the inequalities are in fact strict.

Lemma 2.8. With the notations of (11) and Lemma 2.4, if $g^{\prime}(m)=g(m)-(-1)^{d} g(-m)$, then

$$
g(0)+\sum_{m \geq 1} g^{\prime}(m)=\frac{a(t)}{(1-t)^{d}} .
$$


Proof. It is a standard fact (see, e.g., [4, Exercise 4.6]) that if $G(t)=\sum_{m \geq 0} g(m) t^{m}$, then $\sum_{m \geq 1} g(-m) t^{m}=$ $-G\left(t^{-1}\right)$. Since $G(t)=\frac{h(t)}{(1-t)^{d+1}}+g(0)-h_{0}$, we see that $\sum_{m \geq 1} g(-m) t^{m}=h_{0}-g(0)-\frac{(-t)^{d+1} h\left(t^{-1}\right)}{(1-t)^{d+1}}$. We compute, using Remark 2.5, $g(0)+\sum_{m \geq 1} g^{\prime}(m)=\frac{h(t)}{(1-t)^{d+1}}+(-1)^{d} \frac{(-t)^{d+1} h\left(t^{-1}\right)}{(1-t)^{d+1}}=\frac{a(t)}{(1-t)^{d}}$.

Lemma 2.9. With the notations of (11) and Lemma 2.4, $g_{d} \geq \frac{1}{d !}$. Furthermore, if $h_{0}+\cdots+h_{i} \geq h_{d+1}+$ $\cdots+h_{d+1-i}$ for $0 \leq i \leq\left\lfloor\frac{d}{2}\right\rfloor$, with at least one of these inequalities strict, then $g_{d-1} \geq \frac{1}{2(d-1) !}$.

Proof. By assumption, $g_{d}=\frac{1}{d !} \sum_{i=0}^{d+1} h_{i}$ is positive and hence bounded below by $\frac{1}{d !}$. One verifies that the coefficient of $m^{d-1}$ in $\left(\begin{array}{c}m+d-i \\ d\end{array}\right)$ is $\frac{d+1-2 i}{2(d-1) !}$ and hence $g_{d-1}=\sum_{i=0}^{d+1} h_{i} \frac{d+1-2 i}{2(d-1) !}$. By assumption, we have $h_{0}+\cdots+h_{i} \geq h_{d+1}+\cdots+h_{d+1-i}$ for $0 \leq i \leq d$, with at least one of these inequalities strict. Summing these inequalities gives $\sum_{i=0}^{d+1}(d+1-i) h_{i} \geq\left(\sum_{i=0}^{d+1} i h_{i}\right)+1$ and we conclude that $g_{d-1}=\sum_{i=0}^{d+1} h_{i} \frac{d+1-2 i}{2(d-1) !} \geq$ $\frac{1}{2(d-1) !}$.

Remark 2.10. It follows from the proof of Lemma 2.9 and (2) that if $a(t)$ has degree $d$ and positive integer coefficients, then $g_{d-1} \geq \frac{d+1}{2(d-1) !}$. If $P$ is a $d$-dimensional lattice polytope and $h(t)=\delta_{P}(t)$, then, by Examples 2.3 and 2.7. we recover the well-known fact that the normalised surface area of $P$ is at least $\frac{d+1}{(d-1) !}$.

In the case when $P$ is a $d$-dimensional lattice polytope and $h(t)=\delta_{P}(t)$, the existence of the following inequalities was suggested by Betke and McMullen in [5].

Theorem 2.11. With the notation of (1), if $h_{0}+\cdots+h_{i} \geq h_{d+1}+\cdots+h_{d+1-i}$ for $0 \leq i \leq\left\lfloor\frac{d}{2}\right\rfloor$, with at least one of these inequalities strict, then

$$
g_{d-1-2 r} \leq S_{d-1-2 r}(d-1) g_{d-1}-\frac{\left(h_{0}-h_{d+1}\right) S_{d-2 r}(d-1)}{2(d-2) !} \quad \text { for } \quad 1 \leq r \leq\left\lfloor\frac{d-1}{2}\right\rfloor .
$$

Proof. By Remark 2.6 and Lemma 2.9, the polynomial $g^{\prime}(m)=g(m)-(-1)^{d} g(-m)$ has degree $d-1$ and positive leading coefficient and, by Lemma 2.8 $g(0)+\sum_{m \geq 1} g^{\prime}(m) t^{m}=\frac{a(t)}{(1-t)^{d}}$. Since the coefficients of $a(t)$ are nonnegative by Remark 2.6, applying Theorem 2.2 to $a(t)$ yields inequalities on $g^{\prime}(t)$ and hence on $g(t)$, namely, $g_{d-1-2 r} \leq S_{d-1-2 r}(d-1) g_{d-1}-\frac{a_{0} S_{d-2 r}(d-1)}{2(d-2) !}$ for $1 \leq r \leq\left\lfloor\frac{d-1}{2}\right\rfloor$, where $a_{0}=h_{0}-h_{d+1}$ by (2).

Example 2.12. If $P$ is a $d$-dimensional lattice polytope and $h(t)=\delta_{P}(t)$, then the assumptions of the above theorem hold by Remark 2.6] and Example 2.7, and hence we can bound the coefficients $c_{d-1-2 i}$ in terms of $d$ and the normalised surface area $2 c_{d-1}$ of $P$ (recalling Example 2.3). Betke and McMullen remark in [5] that there are examples showing that a similar bound for $c_{d-2}$ in terms of $d$ and $c_{d-1}$ does not exist.

\section{The Action of $\mathrm{U}_{n}$ on Integer Polynomials}

We will continue with the notation of the previous section and assume from now on that $h_{0}=1$ and that $h(t)$ has degree at most $d$, so that

$$
\sum_{m \geq 0} g(m) t^{m}=\frac{h(t)}{(1-t)^{d+1}}
$$

Fix a positive integer $n$, and recall that $\mathrm{U}_{n} h(t)$ is the polynomial of degree at most $d$ with integer coefficients satisfying $\sum_{m \geq 0} g(n m) t^{m}=\frac{\mathrm{U}_{n} h(t)}{(1-t)^{d+1}}$. We will write $\mathrm{U}_{n} h(t)=h_{0}(n)+h_{1}(n) t+\cdots+h_{d}(n) t^{d}$. The goal of this section is to describe the behaviour of $\mathrm{U}_{n} h(t)$ for sufficiently large $n$. 
Example 3.1. If $P$ is a $d$-dimensional lattice polytope and we set $g(m)=f_{P}(m)$, then, with the notation of the introduction, $h(t)=\delta_{P}(t), \mathrm{U}_{n} h(t)=\delta_{n P}(t)$ and $h_{i}(n)=\delta_{i}(n)$.

The following well-known lemma should be compared with [6. Theorem 1.1].

Lemma 3.2. If $\mathrm{E}_{n}$ is the linear operator that takes a polynomial as input, discards its terms with powers that are not divisible by $n$, and divides each remaining power by $n$, then

$$
\mathrm{U}_{n} h(t)=\mathrm{E}_{n}\left(h(t)\left(1+t+\cdots+t^{n-1}\right)^{d+1}\right) .
$$

Proof. We extend $E_{n}$ to an operator on power series: given a degree- $d$ polynomial $h$, construct the polynomial $g$ such that $\sum_{m \geq 0} g(m) t^{m}=\frac{h(t)}{(1-t)^{d+1}}$. Applying $E_{n}$ to this rational generating function gives

$$
\sum_{m \geq 0} g(n m) t^{m}=\mathrm{E}_{n}\left(\frac{h(t)}{(1-t)^{d+1}}\right)=\mathrm{E}_{n}\left(\frac{h(t)\left(1+t+\cdots+t^{n-1}\right)^{d+1}}{\left(1-t^{n}\right)^{d+1}}\right)=\frac{\mathrm{E}_{n}\left(h(t)\left(1+t+\cdots+t^{n-1}\right)^{d+1}\right)}{(1-t)^{d+1}} .
$$

It follows from the definition that $h_{1}(n)=g(n)-(d+1)$ is a polynomial in $n$ of degree $d$ with positive leading coefficient. Our next goal will be to show that $h_{i}(n)$ is a polynomial in $n$ of degree $d$ with positive leading coefficient for $1 \leq i \leq d$. Now we recall the Eulerian numbers $A(d, i)$ from the introduction; they are positive and symmetric in the sense that $A(d, i)=A(d, d+1-i) \geq 1$ for $1 \leq i \leq d$ [12, p. 242]. The nonzero roots of the Eulerian polynomial $A_{d}(t)=\sum_{i=1}^{d} A(d, i) t^{i}$ are real and negative [12, p. 292, Exercise 3], and consequently we have

$$
\begin{gathered}
A(d, i)^{2}>A(d, i-1) A(d, i+1) \text { for } 2 \leq i \leq d-1 \\
1=A(d, 1)<A(d, 2)<\cdots<A\left(d,\left\lfloor\frac{d+1}{2}\right\rfloor\right), \\
1=A(d, d)<A(d, d-1)<\cdots<A\left(d,\left\lfloor\frac{d}{2}\right\rfloor+1\right) .
\end{gathered}
$$

If we set $g(m)=m^{d}$, then $h(t)=(1-t)^{d+1} \sum_{m \geq 0} m^{d} t^{m}=A_{d}(t)$ [12, p.244] and $\mathrm{U}_{n} A_{d}(t)=(1-$ $t)^{d+1} \sum_{m \geq 0}(n m)^{d} t^{m}=n^{d} A_{d}(t)$. With the convention that $A_{0}(t)=1$, we deduce the following lemma.

Lemma 3.3. If $g(m)=\sum_{j=0}^{d} g_{j} m^{j}$ then $\mathrm{U}_{n} h(t)=\sum_{j=0}^{d} g_{j} A_{j}(t)(1-t)^{d-j} n^{j}$, for every positive integer $n$. In particular, for $1 \leq i \leq d, h_{i}(n)$ is a polynomial in $n$ of degree $d$ of the form

$$
h_{i}(n)=A(d, i) g_{d} n^{d}+(A(d-1, i)-A(d-1, i-1)) g_{d-1} n^{d-1}+O\left(n^{d-2}\right) .
$$

Proof. We compute

$$
\mathrm{U}_{n} h(t)=(1-t)^{d+1} \sum_{m \geq 0} g(n m) t^{m}=(1-t)^{d+1} \sum_{j=0}^{d} g_{j} n^{j} \sum_{m \geq 0} m^{j} t^{m}=\sum_{j=0}^{d}(1-t)^{d-j} g_{j} n^{j} A_{j}(t),
$$

and the second statement follows.

By Lemma 3.3 and the strict log concavity and strict unimodality of the Eulerian numbers, the integers $h_{i}(n)$ are strictly log concave and strictly unimodal for $n$ sufficiently large. Moreover, by the symmetry of the Eulerian numbers, $h_{i+1}(n)-h_{d-i}(n)=2(A(d-1, i)-A(d-1, i-1)) g_{d-1} n^{d-1}+O\left(n^{d-2}\right)$. Hence, if $g_{d-1}>0$ then the strict unimodality of the Eulerian numbers implies that $h_{i+1}(n)>h_{d-i}(n)$ for $n$ sufficiently large and $0 \leq i \leq\left\lfloor\frac{d}{2}\right\rfloor-1$. In a similar direction, Brenti-Welker's Theorem 1.1 says that for $n$ sufficiently large, 
$\mathrm{U}_{n} h(t)$ has negative real roots. We will now consider the existence of bounds for such $n$. We will use the following result of Cauchy (see, for example, [21, Chapter VII]).

Lemma 3.4. Let $p(n)=p_{d} n^{d}+p_{d-1} n^{d-1}+\cdots+p_{0}$ be a polynomial of degree $d$ with real coefficients. The complex roots of $p(n)$ lie in the open disc

$$
\left\{z \in \mathbb{C}:|z|<1+\max _{0 \leq j \leq d}\left|\frac{p_{j}}{p_{d}}\right|\right\}
$$

We are now ready to prove our main result. Our method of proof should be compared with the proof of [3. Theorem 1.2(a)], which gives a bound on the norm of the roots of the Ehrhart polynomial of a lattice polytope, and the proof of [7, Lemma 4.7].

Proof of Theorem 1.2. By Lemma 3.3, $\mathrm{U}_{n} h(t)=n^{d} g_{d}\left(A_{d}(t)+\sum_{j=0}^{d-1} \frac{g_{j}}{g_{d} n^{d-j}} A_{j}(t)(1-t)^{d-j}\right)$. Fix $0<\epsilon \ll$ 1 and let $M_{i}=\max _{t \in\left[\rho_{i}, \rho_{i}+\epsilon\right]}\left|A_{d}(t)\right|$ and $M_{i}^{\prime}=\max _{t \in\left[\rho_{i}-\epsilon, \rho_{i}\right]}\left|A_{d}(t)\right|$ for $1 \leq i \leq d$. Since $g_{d} \geq \frac{1}{d !}$ by Lemma 2.9] and $\left|S_{j}(d)\right| \leq d$ !, Theorem 2.2 implies that

$$
\left|\frac{g_{j}}{g_{d}}\right| \leq\left|(-1)^{d-j} S_{j}(d)+(-1)^{d-j-1} \frac{S_{j+1}(d)}{g_{d}(d-1) !}\right| \leq d !+d ! d,
$$

for $1 \leq j \leq d-1$. Hence, there exists a positive integer $N=N(d, \epsilon)$ such that if $n \geq N(d, \epsilon)$, then

$$
\max _{t \in\left[\rho_{i}, \rho_{i}+\epsilon\right]}\left|\sum_{j=0}^{d-1} \frac{g_{j}}{g_{d} n^{d-j}} A_{j}(t)(1-t)^{d-j}\right|<M_{i} \quad \text { and } \quad \max _{t \in\left[\rho_{i}-\epsilon, \rho_{i}\right]}\left|\sum_{j=0}^{d-1} \frac{g_{j}}{g_{d} n^{d-j}} A_{j}(t)(1-t)^{d-j}\right|<M_{i}^{\prime},
$$

for $1 \leq i \leq d$. Since $\rho_{i}$ is a simple root of $A_{d}(t)$, it follows that for $1 \leq i \leq d$, there exists $t_{i} \in\left[\rho_{i}, \rho_{i}+\epsilon\right]$ and $t_{i}^{\prime} \in\left[\rho_{i}-\epsilon, \rho_{i}\right]$ such that $\mathrm{U}_{n} h\left(t_{i}\right) \neq 0$ and $\mathrm{U}_{n} h\left(t_{i}^{\prime}\right) \neq 0$ have different signs. Observe that since $\mathrm{U}_{n} h(0)=h_{0}>0$, we may and will set $t_{d}=\rho_{d}=0$. We conclude that, for $n \geq N(d, \epsilon)$, we may choose $\beta_{i}(n) \in\left(t_{i}^{\prime}, t_{i}\right)$ and the first assertion follows. Note that if $\mathrm{U}_{n} h(t)$ has negative real roots, then it follows that the coefficients of $\mathrm{U}_{n} h(t)$ are positive, strictly log concave and strictly unimodal.

By Lemma 3.3 , if we set $m_{d}=A\left(d,\left\lfloor\frac{d+1}{2}\right\rfloor\right)+1$, then

$$
m_{d} h_{d}(n)-h_{i}(n)=\left(m_{d}-A(d, i)\right) g_{d} d+\sum_{j=0}^{d-1} \lambda_{j}(d) g_{j} n^{j}
$$

for $0 \leq i \leq d$, where $\lambda_{j}(d)$ is a function of $d$, for $0 \leq j \leq d-1$. By the strict unimodality of the Eulerian numbers, $m_{d} h_{d}(n)-h_{i}(n)$ is a polynomial of degree $d$ with positive leading term. It follows from Lemma 3.4 and (44) that we can bound the absolute value of the roots of $m_{d} h_{d}(n)-h_{i}(n)$ in terms of $d$, and we deduce the second assertion. Similarly, we can bound the absolute values of the roots of $h_{d-i}(n)-h_{i}(n)$ in terms of $d$ for $0 \leq i \leq\left\lfloor\frac{d-1}{2}\right\rfloor$.

Now assume that $h_{0}+\cdots+h_{i+1} \geq h_{d}+\cdots+h_{d-i}$ for $0 \leq i \leq\left\lfloor\frac{d}{2}\right\rfloor-1$. By Lemma 3.3 and the symmetry of the Eulerian numbers,

$$
h_{i+1}(n)-h_{d-i}(n)=2(A(d-1, i+1)-A(d-1, i)) g_{d-1} n^{d-1}+2 \sum_{r=1}^{\left\lfloor\frac{d-1}{2}\right\rfloor} \lambda_{d-1-2 r}(d) g_{d-1-2 r} n^{d-1-2 r},
$$


for $0 \leq i \leq\left\lfloor\frac{d}{2}\right\rfloor-1$, where $\lambda_{d-1-2 r}(d)$ is a function of $d$. By Lemma 2.9 $g_{d-1} \geq \frac{1}{2(d-1) !}$ and hence by Theorem 2.11, we can bound the ratios $\left|g_{d-1-2 r} / g_{d-1}\right|$ in terms of $d$. The above argument then shows that we can bound the absolute values of the roots of $h_{i+1}(n)-h_{d-i}(n)$ in terms of $d$.

Example 3.5. If $P$ is a $d$-dimensional lattice polytope and $h(t)=\delta_{P}(t)$, then $\mathrm{U}_{n} \delta_{P}(t)=\delta_{n P}(t)$ and the assumptions of the above theorem hold by Remark 2.6 and Example 2.7. This establishes Corollary 1.3.

\section{IMPROVing ON THE Bounds}

One would like a bound on the integers $n_{d}$ and $m_{d}$ in Theorem 1.2 In this direction, we will now show that for any positive integer $d$ and $n \geq d$, if $h(t)$ satisfies certain inequalities, then $h_{i+1}(n)>h_{d-i}(n)$ for $i=0, \ldots,\left\lfloor\frac{d}{2}\right\rfloor-1$.

We will continue with the notation of the previous section and consider a polynomial $h(t)$ of degree at most $d$ with integer coefficients. By Lemma 2.4 $h(t)$ has a unique decomposition $h(t)=a(t)+b(t)$, where $a(t)$ and $b(t)$ are polynomials with integer coefficients satisfying $a(t)=t^{d} a\left(\frac{1}{t}\right)$ and $b(t)=t^{d+1} b\left(\frac{1}{t}\right)$. Recall from Remark 2.6 that the coefficients of $a(t)$ are strictly unimodal if and only if $h_{i+1}>h_{d-i}$ for $0 \leq i \leq\left\lfloor\frac{d}{2}\right\rfloor-1$. By Lemma 3.2, for any positive integer $n, \mathrm{U}_{n} h(t)=\mathrm{E}_{n}\left(h(t)\left(1+t+\cdots+t^{n-1}\right)^{d+1}\right)$. Setting $\tilde{a}(t):=$ $\mathrm{E}_{n}\left(a(t)\left(1+t+\cdots+t^{n-1}\right)^{d+1}\right)$ and $\tilde{b}(t):=\mathrm{E}_{n}\left(b(t)\left(1+t+\cdots+t^{n-1}\right)^{d+1}\right)$, we have $\mathrm{U}_{n} h(t)=\tilde{a}(t)+\tilde{b}(t)$. On the other hand, by Lemma 2.4, we have a decomposition, $\mathrm{U}_{n} h(t)=a^{\prime}(t)+b^{\prime}(t)$, where $a^{\prime}(t)$ and $b^{\prime}(t)$ are polynomials with integer coefficients satisfying $a^{\prime}(t)=t^{d} a^{\prime}\left(\frac{1}{t}\right)$ and $b^{\prime}(t)=t^{d+1} b^{\prime}\left(\frac{1}{t}\right)$. Our next goal is to express the polynomials $a^{\prime}(t)$ and $b^{\prime}(t)$ in terms of the polynomials $\tilde{a}(t)$ and $\tilde{b}(t)$. The next lemma says that $\tilde{b}(t)$ only contributes to $b^{\prime}(t)$.

Lemma 4.1. The polynomial $\tilde{b}(t)$ satisfies $\tilde{b}(t)=t^{d+1} \tilde{b}\left(\frac{1}{t}\right)$.

Proof. If we let $f(t)=b(t)\left(1+t+\cdots+t^{n-1}\right)^{d+1}$, then $f(t)=t^{n(d+1)} f\left(\frac{1}{t}\right)$. Applying the operator $\mathrm{E}_{n}$ to both sides gives $\tilde{b}(t)=t^{d+1} \tilde{b}\left(\frac{1}{t}\right)$.

If we use the notation

$$
p(t)=a(t)\left(1+t+\cdots+t^{n-1}\right)^{d+1}=\sum_{k=0}^{n(d+1)-1} p_{k} t^{k}, \quad \text { then } \quad \tilde{a}(t)=\mathrm{E}_{n} p(t) .
$$

Observe that the symmetry of $a(t)$ implies that $p(t)=t^{n(d+1)-1} p\left(\frac{1}{t}\right)$. Hence we may write

$$
p(t)=p_{0}+p_{1} t+\cdots+p_{n} t^{n}+\cdots+p_{n-1} t^{n d}+\cdots+p_{1} t^{n(d+1)-2}+p_{0} t^{n(d+1)-1},
$$

which implies

$$
\tilde{a}(t)=p_{0}+p_{n} t+p_{2 n} t^{2}+\cdots+p_{\left\lfloor\frac{d}{2}\right\rfloor n} t^{\left\lfloor\frac{d}{2}\right\rfloor}+p_{\left\lfloor\frac{d+1}{2}\right\rfloor n-1} t^{\left\lfloor\frac{d}{2}\right\rfloor+1}+\cdots+p_{2 n-1} t^{d-1}+p_{n-1} t^{d} .
$$

With the notation $a^{\prime}(t)=a_{0}^{\prime}+a_{1}^{\prime} t+\cdots+a_{d}^{\prime} t^{d}$, we deduce the following lemma.

Lemma 4.2. For $i=0, \ldots,\left\lfloor\frac{d}{2}\right\rfloor, a_{i}^{\prime}=p_{0}+p_{n}+\cdots+p_{\text {in }}-p_{n-1}-p_{2 n-1}-\cdots-p_{\text {in-1 }}$.

Proof. By Lemma 4.1, to determine $a^{\prime}(t)$ we only need to decompose $\tilde{a}(t)$ into its symmetric components as in Lemma 2.4. The result now follows from (2) and (6). 
If we fix $1 \leq k \leq\left\lfloor\frac{d}{2}\right\rfloor$, then Lemma 4.2 implies that $a_{k}^{\prime}-a_{k-1}^{\prime}=p_{k n}-p_{k n-1}$. If $\gamma_{i}$ denotes the coefficient of $t^{i}$ in $\left(1+t+\cdots+t^{n-1}\right)^{d+1}$, then, $p_{j}=\sum_{i=0}^{d} a_{i} \gamma_{j-i}$ for $j=0, \ldots, n(d+1)-1$, and we conclude that

$$
a_{k}^{\prime}-a_{k-1}^{\prime}=\sum_{i=0}^{d} a_{i}\left(\gamma_{k n-i}-\gamma_{k n-1-i}\right) \text {. }
$$

Lemma 4.3. The coefficients $\left\{\gamma_{i}\right\}$ of $\left(1+t+\cdots+t^{n-1}\right)^{d+1}$ are positive, symmetric and strictly unimodal.

Proof. The lemma follows from the fact that the product of two polynomials with positive, symmetric, unimodal coefficients has positive, symmetric, strictly unimodal coefficients.

By Example 2.7, the assumption in the following lemma holds when $P$ is a $d$-dimensional lattice polytope and $h(t)=\delta_{P}(t)$.

Lemma 4.4. Suppose that $a(t)$ has positive integer coefficients and fix $1 \leq k \leq\left\lfloor\frac{d}{2}\right\rfloor$. If either $n$ and $d$ are even and $n \geq \frac{d}{d+1-2 k}$ or $n \geq \frac{d+1}{d+1-2 k}$, then $a_{k}^{\prime}>a_{k-1}^{\prime}$.

Proof. By Lemma4.3, if $k n \leq\left\lceil\frac{(n-1)(d+1)}{2}\right\rceil$ then $\gamma_{k n-i}-\gamma_{k n-1-i} \geq 0$ for $i=0, \ldots,\left\lfloor\frac{d}{2}\right\rfloor$. Since the coefficients of $a(t)$ are positive, the right hand side of (7) is positive, provided that $k n \leq\left\lceil\frac{(n-1)(d+1)}{2}\right\rceil$. If $n$ and $d$ are even, the latter condition holds if and only if $n \geq \frac{d}{d+1-2 k}$. Otherwise, the condition holds if and only if $n \geq \frac{d+1}{d+1-2 k}$.

Remark 4.5. A similar lemma holds if we only assume that $a(t)$ is nonzero with nonnegative coefficients.

We now prove the main result of this section.

Theorem 4.6. Fix a positive integer $d$ and set $n_{d}=d$ if $d$ is even and $n_{d}=\frac{d+1}{2}$ if $d$ is odd. If $h(t)$ is a polynomial of degree at most $d$ as in (3) satisfying $h_{0}+\cdots+h_{i+1}>h_{d}+\cdots+h_{d-i}$ for $0 \leq i \leq\left\lfloor\frac{d}{2}\right\rfloor-1$, then $h_{i+1}(n)>h_{d-i}(n)$ for $0 \leq i \leq\left\lfloor\frac{d}{2}\right\rfloor-1$ and $n \geq n_{d}$.

Proof. By Remark 2.6 we have assumed that $a(t)$ has positive integer coefficients and we need to show that the polynomial $a^{\prime}(t)$ is strictly unimodal. The result now follows from Lemma 4.4 .

Example 4.7. If $P$ is a $d$-dimensional lattice polytope and $h(t)=\delta_{P}(t)$, then the assumptions of the above theorem hold by Example 2.7

\section{Open Questions}

The main problem that remains concerns optimal choices (beyond Theorem 4.6) for the integers $m_{d}$ and $n_{d}$ in Theorem 1.2 and Corollaries 1.3 and 1.4 . We offer the following conjecture.

Conjecture 5.1. If $\operatorname{dim} P=d$ then $\delta_{n P}(t)$ has distinct, negative real roots for $n \geq d$.

This conjecture holds for $d=2$, by the following argument: A polynomial $1+a_{1} t+a_{2} t^{2}$ has distinct real roots if and only if the discriminant $a_{1}^{2}-4 a_{2}=\left(a_{1}-2\right)^{2}+4\left(a_{1}-a_{2}-1\right)>0$. Hence the polynomial has distinct, real roots if $a_{1}>a_{2}+1$. If $\delta_{n P}(t)=1+h_{1}(n) t+h_{2}(n) t^{2}$, then $h_{1}(n)-h_{2}(n)$ equals the number of lattice points on the boundary of $n P$ minus 3 (see, e.g., [4, Corollary $3.16 \&$ Exercise 4.7]). If $n \geq 2$, each 
edge of $n P$ contains a lattice point that is not a vertex and hence $h_{1}(n)-h_{2}(n) \geq 3$. Thus $h_{1}(n)>h_{2}(n)+1$ and $\delta_{n P}(t)$ has real roots. These roots have to be negative because the coefficients of $\delta_{n P}(t)$ are nonnegative.

Note that an example of a polytope $P$ such that $\delta_{P}(t)$ has complex roots is given by convex hull of $(0,1)$, $(1,0)$ and $(-1,-1)$, with $\delta_{P}(t)=1+t+t^{2}$.

\section{REFERENCES}

1. Christos A. Athanasiadis, $h^{*}$-vectors, Eulerian polynomials and stable polytopes of graphs, Electron. J. Combin. 11 (2004/06), no. 2, Research Paper 6, 13 pp. (electronic).

2. Jörgen Backelin, On the rates of growth of the homologies of Veronese subrings, Algebra, algebraic topology and their interactions (Stockholm, 1983), Lecture Notes in Math., vol. 1183, Springer, Berlin, 1986, pp. 79-100.

3. M. Beck, J. A. De Loera, M. Develin, J. Pfeifle, and R. P. Stanley, Coefficients and roots of Ehrhart polynomials, Integer points in polyhedra - geometry, number theory, algebra, optimization, Contemp. Math., vol. 374, Amer. Math. Soc., Providence, RI, 2005, pp. 15-36.

4. Matthias Beck and Sinai Robins, Computing the continuous discretely, Undergraduate Texts in Mathematics, Springer, New York, 2007.

5. U. Betke and P. McMullen, Lattice points in lattice polytopes, Monatsh. Math. 99 (1985), no. 4, 253-265.

6. Francesco Brenti and Volkmar Welker, The Veronese construction for formal power series and graded algebras, arXiv:0712.2645, 2007, to appear in Adv. Appl. Math.

7. — $f$-vectors of barycentric subdivisions, Math. Z. 259 (2008), no. 4, 849-865.

8. Winfried Bruns and Joseph Gubeladze, Polytopes, rings and K-theory, Springer, to appear.

9. _ Unimodular covers of multiples of polytopes, Doc. Math. 7 (2002), 463-480 (electronic).

10. Winfried Bruns, Joseph Gubeladze, and Ngô Viêt Trung, Normal polytopes, triangulations, and Koszul algebras, J. Reine Angew. Math. 485 (1997), 123-160.

11. Winfried Bruns and Jürgen Herzog, Cohen-Macaulay rings, 2nd ed., Cambridge Studies in Advanced Mathematics, vol. 39, Cambridge University Press, Cambridge, 2008.

12. Louis Comtet, Advanced combinatorics, enlarged ed., D. Reidel Publishing Co., Dordrecht, 1974.

13. Eugène Ehrhart, Sur les polyèdres rationnels homothétiques à n dimensions, C. R. Acad. Sci. Paris 254 (1962), $616-618$.

14. David Eisenbud, Alyson Reeves, and Burt Totaro, Initial ideals, Veronese subrings, and rates of algebras, Adv. Math. 109 (1994), no. 2, 168-187.

15. Juan B. Gil and Sinai Robins, Hecke operators on rational functions. I, Forum Math. 17 (2005), no. 4, 519-554.

16. Takayuki Hibi, Some results on Ehrhart polynomials of convex polytopes, Discrete Math. 83 (1990), no. 1, $119-121$.

17. J.-M. Kantor and K. S. Sarkaria, On primitive subdivisions of an elementary tetrahedron, Pacific J. Math. 211 (2003), no. $1,123-155$.

18. G. Kempf, Finn Faye Knudsen, D. Mumford, and B. Saint-Donat, Toroidal embeddings. I, Springer-Verlag, Berlin, 1973, Lecture Notes in Mathematics, Vol. 339.

19. Jeffrey C. Lagarias and Günter M. Ziegler, Bounds for lattice polytopes containing a fixed number of interior points in a sublattice, Canad. J. Math. 43 (1991), no. 5, 1022-1035.

20. Carl W. Lee, Subdivisions and triangulations of polytopes, Handbook of discrete and computational geometry, CRC Press Ser. Discrete Math. Appl., CRC, Boca Raton, FL, 1997, pp. 271-290.

21. Morris Marden, Geometry of polynomials, Second edition. Mathematical Surveys, No. 3, American Mathematical Society, Providence, R.I., 1966.

22. Richard P. Stanley, Decompositions of rational convex polytopes, Ann. Discrete Math. 6 (1980), 333-342.

23. Alan Stapledon, Inequalities and Ehrhart $\delta$-vectors, arXiv:0711.4382 to appear in Trans. Amer. Math. Soc. 
Department of Mathematics, San Francisco State University, San Francisco, CA 94132, U.S.A.

E-mail address: beck@math.sfsu.edu

Department of Mathematics, University of Michigan, Ann Arbor, Mi 48109, U.S.A.

E-mail address: astapldn@umich.edu 